Rechtsmedizin 2021 · 31:261-262

https://doi.org/10.1007/s00194-021-00515-3

Angenommen: 9. Juni 2021

(c) Springer Medizin Verlag $\mathrm{GmbH}$, ein Teil von Springer Nature 2021

\title{
Die 100. Jahrestagung der Deutschen Gesellschaft für Gerichtliche Medizin/ Rechtsmedizin
}

\author{
Johanna Preuß-Wössner $\cdot$ Knut Albrecht ${ }^{2} \cdot$ Matthias Graw $^{3} \cdot$ Stefanie Ritz-Timme ${ }^{4}$ \\ ${ }^{1}$ Institut für Rechtsmedizin, Universitätsklinikum Schleswig-Holstein, Campus Kiel, Kiel, Deutschland \\ ${ }^{2}$ Brandenburgisches Landesinstitut für Rechtsmedizin, Potsdam, Deutschland \\ ${ }^{3}$ Institut für Rechtsmedizin, LMU, München, Deutschland \\ ${ }^{4}$ Institut für Rechtsmedizin, Universitätsklinikum Düsseldorf, Düsseldorf, Deutschland
}

Liebe Leserinnen und Leser,

während die Fachgesellschaft ihr 100-jähriges Bestehen bereits 2004 feierlich begangen hat, findet die 100. Jahrestagung erst in diesem Jahr statt. Wir freuen uns, dass es möglich war, zu diesem Jubiläum mit der AG Geschichte der Rechtsmedizin der DGRM dieses Schwerpunktheft zu gestalten. Die Geschichte der Jahrestagungen unserer Fachgesellschaft weist einige zeitliche Brüche auf, die insbesondere durch die zwei Weltkriege bedingt sind. Die Coronapandemie hat es tatsächlich nicht geschafft, die Chronologie der Tagungen zu unterbrechen - die Weltkriege und die auf sie folgenden, tiefgreifenden politischen und gesellschaftlichen Veränderungen jedoch schon.

\section{\) Die Geschichte der Jahrestagun- gen spiegelt ein ereignisreiches Jahrhundert}

Nach der 1. Jahrestagung 1905 in Meran und der 9. Jahrestagung 1913 dauerte es 7 Jahre, bis nach dem Ende des 1. Weltkrieges und dem Zusammenbruch Deutschlands und Europas in der bis dahin gekannten politischen Ordnung 1920 wieder eine Tagung (die 10. Jahrestagung) stattfand. Bis auf das Jahr 1923 und 1933 in München (Letztere wurde abgesagt, aber interessanterweise mitgezählt) gab es bis zum Jahr 1940 in jedem Jahr eine Tagung. Diese Chronologie ist im ersten Beitrag des Heftes, „,Von Meran bis München - Die Jah- restagungen der Deutschen Gesellschaft für Gerichtliche Medizin/Rechtsmedizin im Spiegel der Geschichte" von Albrecht et al. im historischen Kontext dargestellt.

Nach dem Ende des 2. Weltkriegs wurde die erste "Friedenstagung" 1951 in Berlin abgehalten. Sie fand, noch als gesamtdeutsche Tagung angelegt, in einer politisch völlig veränderten Landschaft nach der Gründung zweier deutscher Staaten und zweier völlig verschiedener, sich zunehmend feindlich gegenüberstehender politischer Systeme statt. Die 40 Jahre währende Zeit der deutschen Teilung von 1949 bis 1989 stellt einen deutlichen Einschnitt in der Fachgeschichte dar, welcher zur Gründung einer zweiten Fachgesellschaft in der DDR führte. Auch diese Fachgesellschaft führte in der Zeit ihres Bestehens 10 Jahrestagungen und zahlreiche Regionaltagungen und Arbeitsgruppensitzungen durch. Diese werden im zweiten Beitrag „Die Tagungen der Gesellschaft für Gerichtliche Medizin der DDR zwischen 1967 und 1990 - Teil der deutsch-deutschen Fachgeschichte?!" von Preuß-Wössner et al. dargestellt und historisch eingeordnet. Herausgearbeitet wird das Trennende, aber auch das Verbindende zwischen Ost und West, da nach den Aussagen von Zeitzeugen die Kontakte nie komplett abrissen. Eine prägende Persönlichkeit der gerichtlichen Medizin des letzten Jahrhunderts, nicht nur im Spannungsfeld zwischen Ost und West, ist unstrittig Otto Prokop (1921-2009), der 30 Jahre lang Ordinarius für gerichtliche Medizin der Hum- 


\section{Editorial}

boldt-Universität Berlin und Direktor des Institutes der Charité war. Diesem Fachvertreter mit Strahlkraft weit über Deutschland hinaus, dessen Geburtstag sich 2021 zum 100. Mal jährt, ist ein separater Beitrag von Geserick und Wirth gewidmet.

\section{》) Die deutsche Teilung stellt einen deutlichen Einschnitt in der Fachgeschichte dar}

Durch die gesamte Wissenschaftsgeschichte, nicht nur die der gerichtlichen Medizin/Rechtsmedizin, zieht sich v.a. in den frühen Jahren eine deutliche Unterrepräsentanz von Frauen. Im Hintergrund stand die Situation von Frauen im Fach, die geprägt wurde durch eine zunächst offene, gesellschaftlich akzeptierte Diskriminierung, z. B. durch den Ausschluss von den Universitäten, durch eine Reduzierung von Frauen auf das begleitende Damenund Rahmenprogramm auf wissenschaftlichen Tagungen (v.a. im Westen) und im Weiteren durch lange mindestens indirekt fehlende Karrierechancen für Frauen in der Medizin. Den Weg der Frauen in der gerichtlichen Medizin/Rechtsmedizin in Führungspositionen bis in die heutige Zeit beleuchtet die Arbeit "Von Elisabeth Nau (1900-1975) bis heute - Frauen in der deutschsprachigen Gerichts-/ Rechtsmedizin" von Kolbe et al.

Die Vielfalt der bisherigen Tagungsorte ab 1951 bis heute im Sinne einer Zeitreise ergibt sich aus der grafischen Übersicht am Ende des Heftes.

Das vorliegende Heft bietet zur Jubiläumstagung einen historischen Überblick über die Geschichte der Jahrestagungen derFachgesellschaften für gerichtliche Medizin/Rechtsmedizin über den Zeitraum eines in der deutschen Geschichte ausgesprochen ereignisreichen Jahrhunderts bis in das neue Jahrtausend.

Wir dürfen gespannt sein, wie sich unser Fach und unsere Fachgesellschaft mit ihren Tagungen im neuen „digitalen Zeitalter" weiterentwickeln werden. Es liegt in unserer Hand - hier sind alle Mitglieder der Deutschen Gesellschaft für Rechtsmedizin eingeladen -, die Zukunft zu gestalten.

\section{Herzlichst}

Prof. Dr. Johanna Preuß-Wössner, Kiel und Lübeck

Prof. Dr. Knut Albrecht, Potsdam

Prof. Dr. Matthias Graw, München

Prof. Dr. Stefanie Ritz-Timme, Düsseldorf, Präsidentin der DGRM

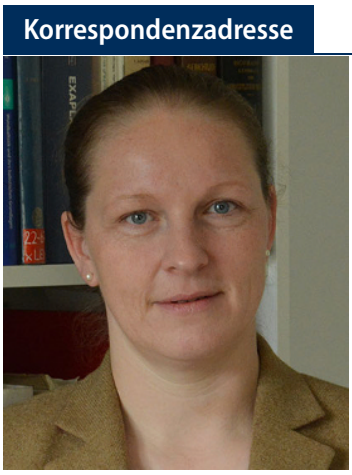

Prof. Dr. Johanna Preuß-Wössner Institut für Rechtsmedizin, Universitätsklinikum Schleswig-Holstein, Campus Kiel Arnold-Heller-Str. 3, 24105 Kiel, Deutschland johanna.preuss-woessner@uksh.de

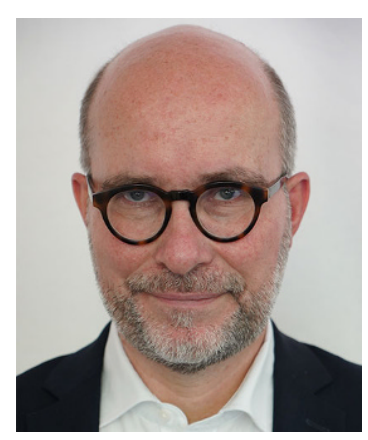

Prof. Dr. Knut Albrecht

Brandenburgisches Landesinstitut für Rechtsmedizin

Lindstedter Chaussee 6, 14469 Potsdam, Deutschland

Knut.Albrecht@BLR.Brandenburg.de

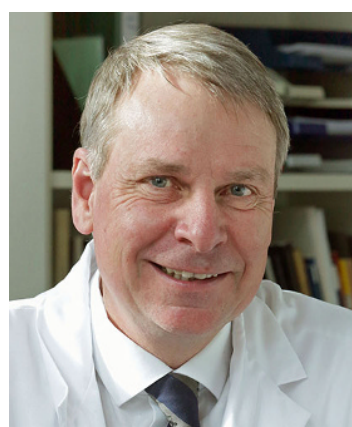

Prof. Dr. Matthias Graw

Institut für Rechtsmedizin, LMU

Nußbaumstr. 26, 80336 München, Deutschland Matthias.Graw@med.uni-muenchen.de

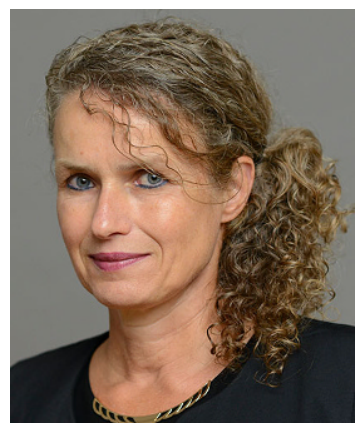

Prof. Dr. Stefanie Ritz-Timme

Institut für Rechtsmedizin, Universitätsklinikum Düsseldorf

Moorenstr. 5, 40225 Düsseldorf, Deutschland Ritz-Timme@med.uni-duesseldorf.de

Interessenkonflikt. J.Preuß-Wössner, K. Albrecht, M. Graw und S. Ritz-Timme geben an, dass kein Interessenkonflikt besteht. 